\title{
New light on Bergman complexes by decomposing matroid types
}

\section{Martin Dlugosch}

Institute of Algebra, Geometry and Topology, University of Bremen, Germany

\begin{abstract}
Bergman complexes are polyhedral complexes associated to matroids. Faces of these complexes are certain matroids, called matroid types, too. In order to understand the structure of these faces we decompose matroid types into direct summands. Ardila/Klivans proved that the Bergman Complex of a matroid can be subdivided into the order complex of the proper part of its lattice of flats. Beyond that Feichtner/Sturmfels showed that the Bergman complex can even be subdivided to the even coarser nested set complex. We will give a much shorter and more general proof of this fact. Generalizing formulas proposed by Ardila/Klivans and Feichtner/Sturmfels for special cases, we present a decomposition into direct sums working for faces of any of these complexes. Additionally we show that it is the finest possible decomposition for faces of the Bergman complex.

Résumé. Les complexes de Bergmann sont des complexes polyhedrales affectés à des matroides. Les faces de ces complexes sont des matroides pour leur part, on les appelle types de matroides. Pour pouvoir comprendre ces types de matroides nous les divisons en sommes directes. Ardila et Klivans ont prouvé que le complexe de Bergman dun matroide peut être subdivisé en le complexe dordre du propre treillis des flats. Au surplus, Feichtner/Sturmfels ont pu montrer que le complexe de Bergmann peut même être subdivisé en le nested set complexe qui est encore plus grossier. Nous y présenterons une preuve plus courte et plus générale. Nous généraliserons des formules qui ont déjà été rédigées pour des cas spéciaux par Ardila/Klivans. Ainsi, nous révélerons une division des types de matroides en sommes directes qui est valable tous les complexes évoqués. De plus, nous montrerons que cette division est la division la plus fine pour les faces du complexe de Bergmann.
\end{abstract}

Keywords: Matroid polytopes, Bergman complexes, Nested set complexes

\section{Introduction}

This is a extended abstract of the paper [Dlu11], which is based on my diploma thesis. For proofs I refer to it.

Let $V$ be a r-dimensional subspace of the n-dimensional vector space $\mathbb{C}^{n}$. The set of vectors

$$
\left(\log \left|v_{1}\right|, \ldots, \log \left|v_{n}\right|\right) \in \mathbb{R}^{n},
$$

for $v_{1}, \ldots, v_{n}$ running through all non-zero elements of $V$, is called the amoeba of $V$. The limit set of these amoebas, for bases of the logarithm approaching zero, is a polyhedral fan called the Bergman fan 
of $V$. It first appeared in the original paper of Bergman [Ber71] as logarithmic limit set of $V$. The study of these spaces is stated as tropical geometry.

Matroid theory comes in when assigning a matroid to $V$ by setting its circuits $C$ as the minimal sets for which there are linear forms of the form $\sum_{i \in C} a_{i} x_{i}$ vanishing on $V$. For introductory references on matroid theory see [Oxl11]. In fact the Bergman fan of $V$ just depends on this associated matroid [Stu02]. It is the set of all vectors $\omega=\left(\omega_{1}, \ldots, \omega_{n}\right) \in \mathbb{R}^{n}$ such that for every circuit $C$ the minimum of the set $\left\{\omega_{i} \mid i \in C\right\}$ is attained at least twice. Although we cut our own path of defining it, from this one we can already see that the Bergman fan is invariant under translation along $\mathbb{R}(1, \ldots, 1)$ and positive scaling. Hence we lose no information when restricting to the sphere $\mathbb{S}=\left\{\omega \in R^{n}: \sum_{i=1}^{n} \omega_{i}=0, \sum_{i=1}^{n} \omega_{i}^{2}=1\right\}$. This restriction is a polyhedral complex called Bergman complex.

Section 2 is devoted to the gathering of current knowledge about matroid polytopes and Bergman fans. Most of it is found in [FS05] and [AK05]. Afterwards we take a look at the concept of nested set complexes by Feichtner and Kozlov [FK04] in Section 3.

The original result of Ardila and Klivans [AK05] is that the Bergman complex of a matroid $M$ can be subdivided to a realisation of the order complex of the proper part of the lattice of flats of $M$. The latter complex is well known [Whi92]. This result was sharpened by Feichtner and Sturmfels [FS05] by the fact that Bergman complexes can even be subdivided to realizations of the even coarser nested set complexes of their respective lattices of flats. Comparing faces of all these complexes by focusing on their vertices, we give a new, much shorter proof of the latter result in Section 4.

Both Ardila/Klivans [AK05, Prop. 2] and Feichtner/Sturmfels [FS05, Thm. 4.4] gave formulas for the supporting matroid types of faces of the order complex respectively the nested set complex in terms of a decomposition into direct sums. In Section 5 we generalize both these formulas giving such a decomposition that even works for faces of the Bergman complex. Taking a closer look we prove that the decomposition for faces of the Bergman complex is the finest one can get i.e. summands are connected.

\section{Matroid polytopes, the Bergman complex and nested sets}

We start with a geometric approach to matroids due to Gel'fand, Goresky, MacPhersen and Serganova [GGMS87]. Let $M$ be a family of subsets of size $r$ of a ground set $\left\{a_{1}, \ldots, a_{n}\right\}$. Each subset $b$ can be represented by its incidence vector in $\mathbb{R}^{n}$, i.e. the $\mathrm{j}$-th coordinate is 1 iff $a_{j} \in b$ and 0 otherwise. Now we can identify $M$ with the convex hull of its elements as incidence vectors:

$$
P_{M}:=\operatorname{conv}\left\{e_{b}: b \in M\right\} .
$$

This yields a convex polytope in $\mathbb{R}^{n}$. Since the generating vertices all lie on the simplex

$$
\Delta:=\left\{\left(x_{1}, \ldots, x_{n}\right) \in \mathbb{R}^{n}: 0 \leq x_{i} \text { for all } i, \sum_{i=1}^{n} x_{i}=r\right\}
$$

the dimension is limited by $n-1$.

Definition 2.1 Let $M$ be a family of r-element subsets of the ground set $\left\{a_{1}, \ldots, a_{n}\right\}$. If every edge of the polytope $P_{M}$ is parallel to $e_{i}-e_{j}$ for some $1 \leq i, j \leq n$ we call $M$ a matroid. The elements of $M$ are called bases of the matroid. The polytope $P_{M}$ is the matroid polytope. 
Let $M$ be a matroid with ground set $E(M)$. A subset $I \subseteq E(M)$ is independent if $I \subseteq b$ for a basis $b \in M$. Hence, bases are the maximal independent sets. If not independent we call a subset of $E(M)$ dependent. The rank of a matroid is simply $r$, the cardinality of its bases. The rank of a subset of $E(M)$ is the cardinality of its largest independent subset. Hence, a set is independent iff its cardinality equals its rank. With circuits we denote minimal dependent sets with respect to inclusion. A flat $F$ of $M$ is a subset such that there is no circuit $c$ of $M$ with $|c \backslash F|=1$. Another way to look at this is that every new element we add to $F$ is increasing the rank. The span of a subset $G$ is the intersection of all flats containing $G$. It is the smallest flat which contains $G$. We can construct it, by adding all the elements of $E(M) \backslash G$ to $G$ which do not increase the rank of $G$. The collection of flats of $M$ can be ordered by inclusion. The resulting poset is a lattice by setting $F_{1} \wedge F_{2}:=F_{1} \cap F_{2}$ and $F_{1} \vee F_{2}:=\operatorname{span}\left(F_{1} \cup F_{2}\right)$. We call it the geometric lattice $\mathcal{L}_{M}$ of the matroid $M$. A matroid $M$ is called loopless if $\bigcup M=\bigcup_{b \in M} b=E(M)$.

Let $F$ and $G$ be flats of $M$ such that $F$ is contained in $G$. We create a new matroid on the ground set $G \backslash F$ by setting:

$$
M[F, G]:=\{b \cap(G \backslash F): b \in M,|b \cap F|=\operatorname{rank}(F),|b \cap G|=\operatorname{rank}(G)\} .
$$

The geometric lattice $\mathcal{L}_{M[F, G]}$ is isomorphic to the interval $[F, G]$ in $\mathcal{L}_{M}$.

There are two special cases of this we want to bring up. The first is $F=\hat{0}$. We will call $M[\hat{0}, G]$ the restriction of $M$ to $G$. Notice that the rank of the matroid $M[\emptyset, G]$ equals $\operatorname{rank}(G)$. The other special case is called contraction and describes the case of $G=\hat{1}=E(M)$. Note that $M[F, E(M)]$ is loopless iff $F$ is a flat of $M$.

There is an equivalence relation on the ground set of a matroid defined as follows: Two elements $x$ and $y$ are equivalent if $x=y$ or there is a circuit of $M$ which contains both $x$ and $y$. The proof that this is an equivalence relation is given in [Oxl11, 124-125], like many other helpful statements for matroids. Note that connected matroids are loopless for ground sets of cardinality of at least 2 . The equivalence classes are the connected components of $M$. Let $c(M)$ denote the number of connected components of $M$. We say a matroid is connected iff $c(M) \leq 1$. The case of $c(M)=0$ belongs solely to the empty matroid which is the matroid with empty ground set.

We say a flat $F$ of $M$ is connected if its restriction $M[\hat{0}, F]$ is connected. Dual to this, it is called co-connected if its contraction $M[F, \hat{1}]$ is connected.

Proposition 2.2 The dimension of the matroid polytope $P_{M}$ is $n-c(M)$.

From here on we are focusing on connected matroids. Every non-connected matroid can be decomposed into a direct sum of connected matroids. Then the matroid polytope of the original matroid is just the product of the matroid polytopes of the direct summands.

Our goal now is to describe the matroid polytope by a system of linear (in-)equalities.

Proposition 2.3 For a connected matroid $M$ of rank $r$ the matroid polytope has the form:

$$
P_{M}=\left\{\left(x_{1}, \ldots, x_{n}\right) \in \Delta: \sum_{i \in F} x_{i} \leq \operatorname{rank}(F) \text { for all flats } F \text { of } M\right\} .
$$

Proof: It is enough to consider the facets of $P_{M}$, since they are bounding the polytope. Let us assume a facet defining inequality is $\sum_{i=1}^{n} a_{i} x_{i} \leq c$, where $n$ is the cardinality of $E(M)$. What we know from 
the definition of matroids is that all the edges are parallel to vectors $e_{i}-e_{j}$. We can compute the normal vector of the facet by looking at the edges it has to be perpendicular to. The constraints the edges impose are all of the form $a_{i}=a_{j}$. Since the normal vector is uniquely determined by these constraints up to scalar multiples we can think of the $a_{i}$ to be either 0 or 1 . So the inequality reduces to $\sum_{i \in A} x_{i} \leq c^{\prime}$ for some $A \subseteq E(M)$ and some $c^{\prime} \in \mathbb{R}$. But what is the maximum the linear form $\sum_{i \in A} x_{i}$ can attain? As a linear form its maximum has to be attained at some face of $P_{M}$. Pick any vertex of this face and evaluate the linear form on it. We obtain that $c^{\prime}=\max \{|b \cap A|: b \in M\}=\operatorname{rank}(A)$. What is left is to show that we just have to pick the subsets $A$ which are flats. Let $\operatorname{span}(A)$ be the flat spanned by $A$. Of course $A \subseteq \operatorname{span}(A)$ and $\operatorname{rank}(A)=\operatorname{rank}(\operatorname{span}(A))$ hold.

$$
\sum_{i \in A} x_{i} \leq \sum_{i \in \operatorname{span}(A)} x_{i} \leq \operatorname{rank}(\operatorname{span}(A))=\operatorname{rank}(A)
$$

So the inequality of $\operatorname{span}(A)$ in the middle already implies the inequality of $A$.

A very important fact, still easy to see, is that every face of $P_{M}$ is a matroid polytope, too, because its edges are still parallel to some difference of standard basis vectors. Assume that, for $\omega \in \mathbb{R}^{n}$, the linear form $\sum_{i=1}^{n} \omega_{i} x_{i}$ attains its maximum in $P_{M}$ at that chosen face. Notice that this linear form can differ from the linear forms in the proposition above. It is not uniquely determined by the chosen face. Considering this we will construct the Bergman complex soon. The bases of this face are exactly the bases of $M$ for which the linear form $\sum_{i=1}^{n} \omega_{i} x_{i}$ attains its maximum. From an algorithmic point of view the bases are exactly the possible outputs of the greedy algorithm with weight $\omega$. The matroid $M_{\omega}$ is called the matroid type of the face.

Since we can represent the matroid polytope $P_{M}$ through a system of linear inequalities indexed by the flats of $M$ we want to filter which of them define facets of the matroid polytope. The question is what combinatorial property do flats have whose linear form attains its maximum at a facet of the matroid polytope. We will call these special flats flacets of $M$.

So the matroid polytope $P_{M}$ is bounded by the inequalities of the flacets and possibly the inequalities of the form $x_{i} \geq 0$. Especially matroids are determined by their flacets.

\section{Proposition 2.4 A flat $F$ of $M$ is a flacet iff it is connected and co-connected.}

The idea of decomposing the matroid type $M_{\omega}$ in as fine of a direct sum as possible is the aim of Section 5 The case of $M_{e_{F}}$ above is a first example of this.

Recall that the linear form $\sum_{i=1}^{n} \omega_{i} x_{i}$ attaining its maximum at a certain face of $P_{M}$ is not uniquely determined. For $c \in \mathbb{R}$ and $c^{+} \in \mathbb{R}^{+}$the linear forms $\sum_{i=1}^{n}\left(\omega_{i} \cdot c^{+}\right) x_{i}$ and $\sum_{i=1}^{n}\left(\omega_{i}+c\right) x_{i}$ induce the same matroid type. So we still get all the different matroid types when restricting $\omega$ to elements of the unit $(n-2)$-sphere contained in a hyperplane orthogonal to $(1, \ldots, 1)$, which is $\mathbb{S}=\left\{\omega \in R^{n}\right.$ : $\left.\sum_{i=1}^{n} \omega_{i}=0, \sum_{i=1}^{n} \omega_{i}^{2}=1\right\}$. The only exception is the matroid type of $\omega=(0, \ldots, 0)$ which is simply $M$ again. Later we will view this as the matroid type of the empty face of the Bergman complex.

Consider the following equivalence relation on $\mathbb{S}: \omega \sim \omega^{\prime}$ iff the induced matroid types $M_{\omega}$ and $M_{\omega^{\prime}}$ coincide. The equivalence classes are relatively open convex polyhedral cones. These cones form a complete fan in $\mathbb{R}^{n}$. It is the normal fan of $P_{M}$. The equivalence classes define a spherical subdivision of $\mathbb{S}$. This subdivision is isomorphic to the boundary of the polar dual $P_{M}^{*}$ of the matroid polytope. For simplicity of notation, we will identify the face of $\partial P_{M}$ just like its dual in $\partial P_{M}^{*}$ with its matroid type $M_{\omega}$. 
Definition 2.5 The Bergman Fan $\widetilde{B}(M)$ is the subfan of the inner fan of $P_{M}$ consisting of the matroid types which are loopless. The Bergman Complex $B(M)$ is the intersection $\widetilde{B}(M) \cap \mathbb{S}$ where $\mathbb{S}=\left\{\omega \in \mathbb{R}^{n}\right.$ : $\left.\sum_{i=1}^{n} \omega_{i}=0, \sum_{i=1}^{n} \omega_{i}^{2}=1\right\}$.

After this geometric realisation let us reduce the Bergman complex to its combinatorial data, i.e. to its face poset. The faces of the Bergman complex $B(M)$ are the matroid types $M_{\omega}$ which are loopless. Since the matroid types are subsets of bases of $M$ they come with the natural partial order of inclusion. The order of the face poset of the Bergman complex is the dual of this order. So the face $M_{\omega}$ is contained in the face $M_{\omega^{\prime}}$ in $B(M)$ iff the reversed inclusion holds for $M_{\omega}$ and $M_{\omega^{\prime}}$ as subsets of $M$.

Let us consider what the vertices of the Bergman complex are. Apart from the empty face they are the minimal faces of $P_{M}^{*}$ i.e. the maximal faces of $P_{M}$, whose matroid types are loopless. But obviously the matroid type of a face of $P_{M}$ is loopless iff the face is not contained in one of the hyperplanes of the form $x_{i}=0$. So the vertices of $B(M)$ are duals of facets of $P_{M}$ whose hyperplanes are not of the form $x_{i}=0$. We already determined what these are, namely the flacets of $M$. Since every face of $P_{M}$ is uniquely determined by the set of facets of the polyhedral complex which contain it, every face of $B(M)$ is uniquely determined by its vertices.

Example 2.6 Let $M$ be the matroid with ground set $\{1,2,3,4,5,6\}$ and circuits $\{1,2,3,4\},\{1,2,5,6\}$ and $\{3,4,5,6\}$. Hence the bases are all subsets of size four except for the circuits. There are two types of flacets here. On the one hand there are the singletons i.e. the subsets of size one. On the other hand there are the three circuits itself.

The Bergman complex is a pure polyhedral complex of dimension two. There are two kinds of facets of the Bergman complex. There are twenty triangles but there are also three quadrangles, whose vertices are shortly notated:

$$
1,2,1234,1256 \quad 3,4,1234,3456 \quad 5,6,1256,3456 .
$$

This shows that the Bergman complex can be non-simplicial.

\section{Nested set complexes}

Beside order complexes this is another way of creating a simplicial complex from a geometric lattice due to Feichtner and Kozlov [FK04].

For a semi-meet lattice $\mathcal{L}$ let intervals in $\mathcal{L}$ be denoted by $[X, Y]:=\{Z \in \mathcal{L}: X \leq Z \leq Y\}$. For any $X \in \mathcal{L}$ and any subset $S \subseteq \mathcal{L}$ write $S_{\leq X}:=\{Y \in S: Y \leq X\}$. The same way we can define $S_{<X}, S_{\geq X}$ and $S_{>X}$. Last but not least, the set of maximal elements in $S \subseteq \mathcal{L}$ is denoted by $\max S$.

Definition 3.1 For a finite lattice $\mathcal{L}$ a subset $\mathcal{G}$ in $\mathcal{L}_{>0}$ is a building set if for any $X \in \mathcal{L}_{>0}$ with $\max \mathcal{G}_{\leq X}=\left\{G_{1}, \ldots, G_{k}\right\}$ the map

$$
\Phi_{X}: \Pi_{j=1}^{k}\left[\hat{0}, G_{j}\right] \longrightarrow[\hat{0}, X]
$$

induced by the inclusions of the intervals $\left[\hat{0}, G_{j}\right] \subseteq[\hat{0}, X]$ is an isomorphism of posets.

In colorful language, the condition means that $X$ can be decomposed into $G_{1}, \ldots, G_{k}$ and the properties of $[\hat{0}, X]$ can be separately investigated in the intervals $\left[\hat{0}, G_{j}\right]$. 
There is always a maximal and a minimal building set. The maximal one is always the whole lattice without $\hat{0}$. In this case $X$ is decomposed into just one factor, $X$ itself. The minimal building set $\mathcal{G}_{\text {min }}$ consists of all connected flats and, if $\mathcal{L}$ is not connected already, the top element $\hat{1}$.

Definition 3.2 Let $\mathcal{L}$ be a finite lattice and $\mathcal{G}$ a building set containing the top element $\hat{1}$. A subset $S \subseteq \mathcal{G}$ is called nested if for any set of incomparable elements $X_{1}, \ldots, X_{k}$ of at least two elements of $S$ the join $X_{1} \vee \ldots \vee X_{k}$ does not lie in the building set $\mathcal{G}$ again. Since subsets of $S$ fulfill the same condition, again this is a simplicial complex. Topologically, it is a cone with apex $\{\hat{1}\}$. Its link $\mathcal{N}(\mathcal{L}, \mathcal{G})$ is the nested set complex of $\mathcal{L}$ with respect to the building set $\mathcal{G}$.

The case of the minimal building set $\mathcal{G}_{\text {min }}$ is called the nested set complex $\mathcal{N}\left(\mathcal{L}, \mathcal{G}_{\text {min }}\right)$. If there is no hint about the building set, it is the minimal one. The other extreme is the maximal building set. Since every join of incomparable elements is an element of the building set the only nested sets are those which are totally ordered. So the simplices are just the chains in the proper part $\mathcal{L}-\{\hat{0}, \hat{1}\}$. Thus the nested set complex with respect to the maximal building set equals the order complex of the proper part of $\mathcal{L}$.

A lattice is atomic if every element is a join of atoms. Our geometric lattices are such atomic lattices. For simple matroids these atoms are just the elements of the ground set $E(M)$. For arbitrary atomic lattices Feichtner and Yuzvinsky [FY04] proposed the following polyhedral realization of nested set complexes.

Let $\mathcal{L}$ be an atomic lattice with atoms $\left\{a_{1}, \ldots, a_{n}\right\}$ and $\mathcal{G}$ a building set containing $\hat{1}$. For any $G \in \mathcal{G}$ let $e_{G} \in \mathbb{R}^{n}$ be the incidence vector of $G$ respective the set of atoms i.e. the i-th coordinate is 1 iff $a_{1} \subseteq G$ and 0 otherwise. For a nested set $S$ the set of incidence vectors of its elements is linearly independent. Hence with $\mathbb{R}_{\geq 0}\left\{e_{G} \mid G \in S\right\}$, they span a simplicial cone. For nested sets $S$ and $S^{\prime}$ they intersect exactly in the cone belonging to the nested set $S \cap S^{\prime}$. Thus the set of cones from nested sets form a simplicial fan.

Just like the Bergman Fan this fan has the property that its cones are invariant under the translation along the line $\mathbb{R}(1, \ldots, 1)$. So again we loose no information when restricting the fan to the (n-2)-sphere $\mathbb{S}=\left\{\omega \in R^{n}: \sum_{i=1}^{n} \omega_{i}=0, \sum_{i=1}^{n} \omega_{i}^{2}=1\right\}$. The resulting spherical complex is a geometric realization of the nested set complex.

In order to compare nested set complexes of different building sets, Feichtner and Müller [FM05] proved that for building sets $\mathcal{G}$ and $\mathcal{G} \cup\{X\}$ the nested set complex respective the building set $\mathcal{G} \cup\{x\}$ can be obtained by a stellar subdivision from the nested set complex of the smaller building set $\mathcal{G}$ at the simplex corresponding to the factors of $X$ respective $\mathcal{G}$. Recursively we can construct the order complex of the proper part $\mathcal{L}-\{\hat{0}, \hat{1}\}$, which is the nested set complex of the maximal building set, from the nested set complex of the minimal building set by a sequence of stellar subdivisions. The single steps correspond to adding elements to the building sets in a non decreasing order. In particular the order complex of the proper part of $\mathcal{L}$ and the nested set complex are homeomorphic.

Example 3.3 Consider the matroid of Example 2.6. The lattice $\mathcal{L}$ is the lattice of flats of the matroid $M$. Though this is not true in general, the minimal building set $\mathcal{G}_{\text {min }}$ consists exactly of the set of flacets. The complexes coincide except for the three squares which are each replaced by two triangles with vertices:

$$
1,2,1234 \quad 1,2,1256 \quad 3,4,1234 \quad 3,4,3456 \quad 5,6,1256 \quad 5,6,3456 .
$$




\section{Comparison of order complex, nested set complex and Bergman complex}

Ardila and Klivans [AK05] first showed that the order complex of proper part of the lattice of flats is a refinement of the Bergman complex. Feichtner and Sturmfels [FS05] proved that this is even true for the nested set complex. Though we can gain a lot of insight from their proof it is a little bit complicated. From another point of view this can be seen easier.

Remember the geometric realizations of the order complex (nested set complex of the maximal building set), the nested set complex (with minimal building set) and the Bergman complex.

What faces of all these complexes have in common is that they are the spherical convex of their vertices. The vertices are the scaled incidence vectors of flats in the case of the order complex, connected flats in the case of the nested set complex and flacets in the case of the Bergman complex.

Theorem 4.1 For any of these complexes any element $\omega$ in the cone corresponding to some face with vertices $\Gamma$ has the form $\sum_{F \in \Gamma} \lambda_{F} \cdot e_{F}$ for all $\lambda_{F} \gtrless 0$. Then identifying the matroid type $M_{\omega}$ with its set of bases:

$$
M_{\omega}=\{b \in M \mid \text { for all } F \in \Gamma:|b \cap F|=\operatorname{rank}(F)\} .
$$

Proof: A basis $b$ of $M$ maximizes the linear functional $\omega$ in $P_{M}$ iff for all bases $b^{\prime}$ of $M$ the inequality $e_{b^{\prime}} \cdot \omega \leq e_{b} \cdot \omega$ holds. First consider a basis $b$ satisfying $|b \cap F|=\operatorname{rank}(F)$ for all $F \in \Gamma$.

$$
\begin{aligned}
e_{b^{\prime}} \cdot \omega & =e_{b^{\prime}} \cdot\left(\sum_{F \in \Gamma} e_{F}\right)=\sum_{F \in \Gamma} e_{b^{\prime}} \cdot e_{F}=\sum_{F \in \Gamma}\left|b^{\prime} \cap F\right| \\
& \leq \sum_{F \in \Gamma} \operatorname{rank}(F)=\sum_{F \in \Gamma}|b \cap F|=\sum_{F \in \Gamma} e_{b} \cdot e_{F}=e_{b} \cdot\left(\sum_{F \in \Gamma} e_{F}\right)=e_{b} \cdot \omega
\end{aligned}
$$

This shows that $b$ is at least a basis of $M_{\omega}$.

Conversely assume $e_{b^{\prime}} \cdot \omega \leq e_{b} \cdot \omega$ always holds. Choose $b^{\prime}$ such that $|b \cap F|=\operatorname{rank}(F)$ holds for all $F \in \Gamma$. Then the equations above teaches us that $\sum_{F \in \Gamma}|b \cap F|=\sum_{F \in \Gamma} \operatorname{rank}(F)$. Since for all pairs of summands the inequality $|b \cap F| \leq \operatorname{rank}(F)$ holds, equality holds for them, too.

Corollary 4.2 For all building sets $\mathcal{G}$ the nested set complex $N\left(\mathcal{L}_{M}, \mathcal{G}\right)$ is a refinement of the Bergman fan.

Proof: Already Ardila and Klivans [AK05, Thm. 1] showed that the $\omega \in \mathbb{R}^{n}$ for which $M_{\omega}$ is loopless are exactly the ones lying in the interior of polyhedral cones spanned by incidence vectors of flats. Due to Theorem 4.1 the induced matroid types are the same for all elements of any face of the realizations of our complexes.

Example 4.3 Consider the matroid of Example 2.6 and 3.3 In Figure 1 we compare the excerpts of the order complex $\Delta(\mathcal{L}-\{\hat{0}, \hat{1}\})$, the nested set complex $\mathcal{N}\left(\mathcal{L}_{M}, G_{\text {min }}\right)$ and the Bergman complex $B(M)$ which arise as subdivisions of each other. The matroid type of the two-dimensional face is $M_{\omega}=\{1235,1236,1245,1246\}$. 


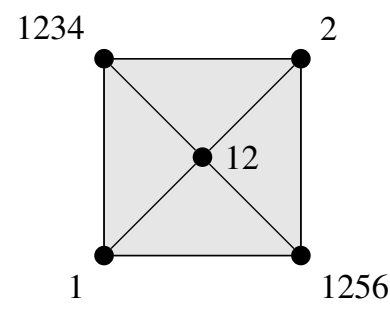

(a) excerpt of $\Delta\left(\mathcal{L}_{M}\right)$

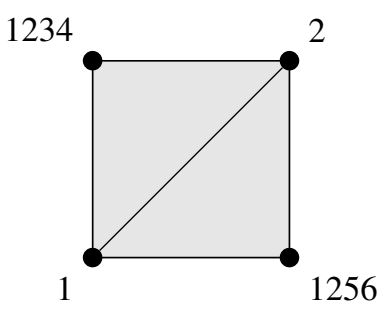

(b) excerpt of $\mathcal{N}\left(\mathcal{L}_{M}, G_{\text {min }}\right)$

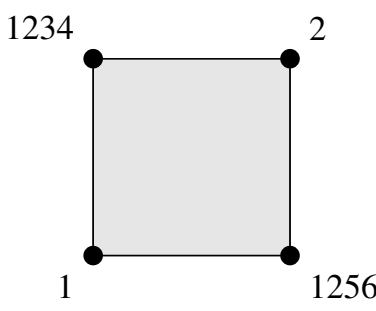

(c) excerpt of $\mathrm{B}(\mathrm{M})$

Figure 1: The same excerpt of all polyhedral complexes

\section{Decomposition of matroid types}

Definition 5.1 Let $A$ be a subset of $E(M)$. We say that $A$ has full $\omega$-rank if for all bases $b \in M_{\omega}$, $|A \cap b|=\operatorname{rank}(A)$ holds.

Note that the vertices of a face have full $\omega$-rank by Theorem 4.1 Here is an immediate application of this important definition.

Proposition 5.2 A matroid type $M_{\omega}$ is loopless iff all sets $A$ with full $\omega$-rank are flats of $M$.

Proposition 5.3 Let $A$ and $B$ both having full $\omega$-rank. Then $A \cap B, A \cup B$ and every connected component of a set with full $\omega$-rank have full $\omega$-rank, too.

With this knowledge we see that the set of subsets with full $\omega$-rank is a sublattice of $\mathcal{L}_{M}$. It has the property that the join is already the union instead of just its span. Additionally it is closed under taking connected components i.e. if an interval $[A, B]$ in $\mathcal{L}_{M}$ is isomorphic to $\left[A, C_{1}\right] \times\left[A, C_{2}\right]$ for $A<C_{1}, C_{2}<B$ and $A, B$ have $\omega$-full rank then both $C_{1}$ and $C_{2}$ have this property, too. All information about $M_{\omega}$ is contained in this special sublattice. It is the sublattice, closed under taking connected components, which is induced by the set of flacets and the elements $\hat{0}, \hat{1}$.

Theorem 5.4 Let $M_{\omega}$ be the matroid type of any face of either the order complex $\Delta\left(\mathcal{L}_{M}\right)$, the nested set complex $\mathcal{N}\left(\mathcal{L}_{M}, G_{\text {min }}\right)$ or the Bergman complex $B(M)$ and $\Gamma$ its set of vertices. Additionally let $\Pi:=\bigvee_{F \in \Gamma}(F \mid E(M)-F)$ be the partition of $E(M)$ which is the join in $\Pi_{E(M)}$ of the partitions consisting of just two blocks, the vertex and its complement. For any block $\alpha$ in $\Pi$, let $\Gamma_{\alpha}$ denote the elements of $\Gamma$ which contain $\alpha$ as a subset. Then,

$$
M_{\omega} \cong \bigoplus_{\alpha \in \Pi} M\left[\bigcap \Gamma_{\alpha} \backslash \alpha, \bigcap \Gamma_{\alpha}\right]
$$

Example 5.5 For the matroid $M$ of Example 2.6 and the face of the Bergman complex with vertices $1,2,1234,1256$, the partition $\Pi$ is $1|2| 34 \mid 56$. Its matroid type decomposition is

$$
M[\emptyset, 1] \oplus M[\emptyset, 2] \oplus M[12,1234] \oplus M[12,1256] .
$$


Corollary 5.6 Let $M$ be a connected matroid. A set of flacets $\Gamma=\left\{F_{1}, \ldots, F_{k}\right\}$, with associated partition $\Pi:=\bigvee_{i}^{k}\left(F_{i} \mid E(M)-F_{i}\right)$, is the set of vertices of a face of $B(M)$ iff the following conditions are fulfilled:

- For all $x$ in $E(M)$ if $x \in \alpha$ then there is at least one basis $b \in M$ s.t. $\left|b \cap \bigcap \Gamma_{\alpha}\right|=\operatorname{rank}\left(\bigcap \Gamma_{\alpha}\right),\left|b \cap \bigcap \Gamma_{\alpha} \backslash \alpha\right|=\operatorname{rank}\left(\bigcap \Gamma_{\alpha} \backslash \alpha\right)$ and $x \in b$.

- There is a basis $b \in M$ such that $|b \cap F|=\operatorname{rank}(F)$ for all $F \in \Gamma$

- there is no other flacet $F^{\prime}$ of $M$ with the property that $\left|b \cap F^{\prime}\right|=\operatorname{rank}\left(F^{\prime}\right)$ for all bases $b \in M$, which fulfill $|b \cap F|=\operatorname{rank}(F)$ for all flacets $F$ of $\Gamma$.

In this case $\bigoplus_{\alpha \in \Pi} M\left[\cap \Gamma_{\alpha} \backslash \alpha, \bigcap \Gamma_{\alpha}\right]$ is the matroid type of the face and $\Gamma$ its set of flacets.

Theorem 5.7 Let $M$ be a connected matroid. For a face of its Bergman complex the decomposition of the matroid type in theorem 5.4 is the finest one can get i.e. the direct summands are connected.

In order to show this, we will use the following propositions in the following way. We start with a purely technical lemma which is made for prooving Proposition 5.9. Together with the latter and Proposition 5.10 one can show Proposition 5.11, which is the final ingrediant for the proof of the Theorem above.

Lemma 5.8 Let $A$ be a connected flat. We denote the connected components of $M[A, E(M)]$ by $K_{1}, \ldots, K_{m}$. Let $c$ be a circuit of $M$ and $x \in c \cap K_{i}$ for a certain $K_{i}$. Then there exists a circuit $c^{\prime}$ of $M$ such that $c^{\prime}-A \subseteq c-A, x \in c^{\prime} \cap K_{i}$ and $c^{\prime}-A$ is a circuit of $M[A, E(M)]$.

Proposition 5.9 Let $A$ be a connected flat of $M$ and $K_{1}, \ldots, K_{t}$ connected components of $M[A, E(M)]$. Then $F_{i}:=A \cup \bigcup_{j \neq i} K_{j}=E(M)-K_{i}$ is a flacet of $M$.

Proposition 5.10 Let $A \subseteq E(M)$ and $A_{1}, \ldots, A_{n}$ connected components of $M[\emptyset, A]$. We denote the connected components of $M[A, E(M)]$ by $K_{1}, \ldots, K_{t}$. If $A$ has full $\omega$-rank, then for any $K_{i}$ and any connected component $G$ of $M\left[\emptyset, A \cup K_{i}\right]$, which intersects $K_{i}$, both $A \cup K_{i}$ and $G$ have full $\omega$-rank, too.

Proposition 5.11 Every connected flat A of a matroid $M$ is an intersection of flacets of $M$. Furthermore, if $A$ has full $\omega$-rank, then so do these flacets. This means they are vertices of the face $M_{\omega}$ of the Bergman complex.

In the end we can compare the subdivision of a face of the order complex with the subdivisions of its supporting faces of the coarser complexes.

Corollary 5.12 The decomposition of the matroid type in 5.4 for a face of the order complex is coarser than the decomposition of the matroid type of its supporting face in the nested set complex.

The decomposition of the matroid type for a face of the nested set complex is coarser than the decomposition of the matroid type of its supporting face in the Bergman complex.

Note that these relations are strict iff the dimension of the faces is increasing while taking the support face in the next coarser complex. So for maximal faces the decompositions are all the same. Using this we can easily see that the maximal faces of the Bergman complex correspond to transversal matroids i.e. direct sums of matroids of rank 1 . 


\section{References}

[AK05] F. Ardila and C.J. Klivans, The Bergman complex of a matroid and phylogenetic trees, J. Combin. Theory Ser. B 96 (2005), no. 1, 38-49.

[Ber71] G.M. Bergman, The logarithmic limit-set of an algebraic variety, Transactions of the American Mathematical Society (1971), 459-469.

[Dlu11] M. Dlugosch, New light on Bergman complexes by decomposing matroid types, arXiv:1111.5496, November 2011.

[FK04] E.M. Feichtner and D.N. Kozlov, Incidence combinatorics of resolutions, Selecta Math. (N.S.) 10 (2004), no. 1, 37-60.

[FM05] E.M. Feichtner and I. Müller, On the topology of nested set complexes, Proc. Amer. Math. Soc. 133 (2005), no. 4, 999-1006.

[FS05] E.M. Feichtner and B. Sturmfels, Matroid polytopes, nested sets and Bergman fans, Port. Math. (N.S.) 52 (2005), no. 4, 437-468.

[FY04] E.M. Feichtner and S. Yuzvinsky, Chow rings of toric varieties defined by atomic lattices, Inventiones Mathematicae 155 (2004), 515-536.

[GGMS87] I.M. Gelfand, R.M. Goresky, R.D. MacPherson, and V.V. Serganova, Combinatorial geometries, convex polyhedra, and Schubert cells, Adv. Math 63 (1987), no. 3, 301-316.

[Ox111] J. Oxley, Matroid theory, Oxford University Press, 2011.

[Stu02] B. Sturmfels, Solving systems of polynomial equations, Amer.Math.Soc., CBMS Regional Conferences Series, No 97, Providence, Rhode Island, 2002.

[Whi92] N. White (ed.), Matroid applications., Encyclopedia of Mathematics and Its Applications. 40. Cambridge: Cambridge University Press. xii, 363 p., 1992. 